\title{
Mental Imagery and Spatio-Temporal Continuity: Evidence from the Function of the Hippocampal Formation
}

\author{
Anna Kocsis \\ Institute of Philosophy, Zagreb, Croatia \\ anna.kocsis1@gmail.com
}

Received 1 July 2018; accepted 8 November 2018; published 17 September 2019

\begin{abstract}
The aim of this paper is to suggest a novel account of mental imagery according to which mental images are not a-temporal picture-like representations, but processes characterized by their spatio-temporal continuity. Evidence based in particular on recent advances in understating the functional role of the hippocampal formation in cognition and spatial coding is provided. Under this account, mental images are a pervasive form of cognition that is supported by the complex interaction of the hippocampus and the entorhinal cortex, encompassing cognitive functions such as navigation, episodic memory, as well as mental rotation and scanning. The functional role of the hippocampus is twofold: it forms elements of spatio-temporal continuity and re-combines them in novel ways in the process of scene reconstruction that underpins various forms of spatial cognition.
\end{abstract}

Keywords: hippocampus; grid cells; mental imagery; place cells; spatio-temporal continuity.

\section{Introduction}

In his Critique of Pure Reason, Kant famously stated that "Space is not something objective and real, $[\ldots]$ instead, it is subjective and ideal, and originates from the mind's nature in accord with a stable law as a scheme, as it were, for coordinating everything sensed externally" (Kant, 1781/1998, p. 403). For Kant, representation of space was a fundamental and innate organizing principle of all cognition - the underpinning of all sensory experience. Thus, the representation of space is not a product of perceiving and interacting in the world but a form that is "enforced" on all experience, and a pre-requisite of conceiving an object or possessing an idea of an object. The organizing role that space has in the 
structures of cognition, i.e. representations, has been at the center of scientific inquiry since the pioneering ideas of O'Keefe (1976), progressing from the discovery of place cells in the hippocampus (O'Keefe \& Dostrovsky, 1971), through the identification of grid cells in the entorhinal cortex (Hafting, Fyhn, Molden, Moser, \& Moser, 2005), and has culminated in a deeper understanding of the fundamental role that the spatio-temporal structure plays in cognition. This has led to the emergence of a unified picture of cognitive processes, such as path integration, episodic memory and mental imagery, which all rely on spatio-temporal information conveyed by the hippocampal formation.

More specifically, state-of-the-art research points to the crucial role of the hippocampus in generating and storing sequences of spatio-temporal continuity (e.g. Burgess, Maguire, \& O'Keefe, 2002; Buzsáki \& Moser, 2013), which are the basic pre-requisites of all spatial cognition. The nature of the information-bearing structures coded in the hippocampal complex and particularly in the hippocampus itself is as yet undetermined. In their pioneering work, O'Keefe \& Nadel (1978) suggested the hippocampus was a "seat" of cognitive maps - a particular information-bearing structure, the existence of which was originally proposed by Tolman (1948) several decades previously. Cognitive maps were suggested to provide a sense of place - the position of one's body in the environment and relative to surrounding objects. Recent research had been able to further develop this original idea and offer a better understanding of the functional role of the hippocampus in various types of cognitive processes that rely on spatial information.

The mental imagery debate, which for a while has been an open issue in cognitive science and philosophy alike, has been from the start in many ways related to the aforementioned question posed by Kant. Mental images as a sui generis form of content-bearing structures were forcefully defended by Kosslyn (e.g. 1981, 1980), who considered mental rotation and scanning as paramount examples of imagistic thinking. These are both spatial processes that unfold in real time and were of specific interest to Kosslyn exactly due to the coupling between the psychological dimensions of space and time that corresponds to their counterparts in physical reality. However, unlike Kant and more along the lines of cartesianism, Kosslyn envisaged a functional characterization of mental imagery. While considering the spatial aspect of mental imagery as its defining feature, Kosslyn did not provide a clear account of the encoding process by which spatial (and temporal) features are organized in a way that supports the functional roles of mental imagery. The aim of this paper is to shed new light on mental imagery in terms of its structural characteristics and prevalence in cognitive life, as revealed by neuro-scientific research into spatial cognition and, more specifically, into the functioning of the hippocampal formation. ${ }^{1}$

\footnotetext{
${ }^{1}$ Philosophical treatments of mental imagery have traditionally been biased by division between the empiricist and rationalist schools of thought. The two dominant characterizations of mental imagery, Kosslyn's quasi-pictorial theory and the description theory advanced by Pylyshyn (e.g., 1973, 1981), both align themselves with the rationalist treatment of cognition by assuming that only an auxiliary role may be granted to mental imagery. According to the champions of the rationalist approach to cognition (e.g. Frege, Wittgenstein, Fodor), the primary units of cognition are language-like vehicles whose semantical characters are not obtained through a process of abstracting
} 
The structure of this paper is as follows: the first section is a brief historical introduction to the roles that spatial organization has played in understanding animal behavior in terms of spatial navigation based on "cognitive maps," as well as in Kosslyn's quasi-pictorial account of mental imagery. The goal of this section is to make overt the similarities in the treatment of space between these separate lines of investigation. The following section provides a brief and concise overview of the main results in the ongoing research into the function of the hippocampal complex. Place and grid cells are introduced, and their roles in path integration and episodic memory are discussed. It is argued that grid cells provide a universal metric for space, in line with Kant's original idea; however, it is also emphasized that the information relayed to the hippocampus results in complex representations whose relation to this grid system is currently not known. In the final section, a hypothesis concerning the functional role of the hippocampus in mental imagery is presented. According to this approach, the hippocampus is a loci of spatio-temporal pattern separation, a process that underlies access to and coding of cognitive maps, path integration, episodic memory and mental imagery. As a conclusion, a new understanding of mental imagery is suggested; mental images are episodes of spatio-temporal continuity that are "chunked" or "grouped" by the activity of the hippocampus. They are elements of a complex spatial structure in whose formation the hippocampus plays a fundamental role in terms of binding the various elements (or chunks) of the mental image together into a consistent spatio-temporal frame.

\section{Space as an Organizing Principle: Spatial Navigation and Mental Imagery}

For any adaptive behavior of an organism, a necessary precondition is finding its way around the world and perceiving the position of its body relative to the surroundings. This feat was in the focus of behaviorists like Pavlov, Watson, Hull, and Skinner, who believed spatial navigation is a form of response learning in which an animal memorizes a series of body movements it has to perform in order to obtain a reward. In other words, there is no open-ended representation of the environment and only the goal-directed instructions for successful behavior are learnt by an animal. Work done by Tolman, Ritchie, and Kalish (1946) contradicted this idea and showed that animals (rats, in this case) were capable of both place learning and response learning, which was received as a radical demonstration of the failures of behaviorism. By placing rats into a maze at different positions relative to the position of the reward (i.e. the decisions about which direction to take in order to reach

\footnotetext{
away from sensorial experiences, i.e. they are not grounded in sensori-motor percepts. In response to this, some authors have put forward theories strongly aligned with the empiricist camp (e.g., Barsalou, 1999; Prinz, 2004). The aim of these approaches is to ground the conceptual system in imagery-like mental representations by reinventing or reshaping the notion of concepts through the introduction of novel sub-types (perceptual symbol system and proxytype theory, respectively). Such treatment of mental imagery essentially places it in the ballpark of the debate around the modal/amodal nature of concepts. However, focusing on the framework defined by the traditional ways of characterizing concepts and conceptual content has a hindering effect on establishing what the unique features and contributions of imagistic cognition are. The aim of this paper is to provide a treatment of mental imagery that is independent of its classical treatment in relation to symbolic conceptual systems.
} 
the reward were being manipulated), Tolman and colleagues were able to show that rats change their decisions according to the geometry of the maze by adopting novel routes to the reward in a way that maximizes the overall amount of food received. Additionally, rats that were required to adapt their decisions were better at reaching the food rewards than rats that simply needed to remember the same sequence of decisions.

This work had remarkable consequences not only on the field of learning research, but also on the scientific understanding of the brain as such; there were at least two reasons for this. First, it shows that animals are capable of model-based learning ${ }^{2}$. Second, the parsimonious explanation of these findings is that the mental models that animals use are of a particular format; namely they are in some way a flexible (i.e. easy to rotate) spatial representation of the environment. Thus, Tolman (1948) himself and others (e.g. Gallistel, 1990; O'Keefe \& Nadel, 1978) suggested that local navigation is supported by internal "cognitive maps" of the layout of the immediate environment, and the exploration of the environment gradually results in a gestalt of the environment. ${ }^{3}$

The characterization of the phrase "cognitive map" comes in many forms (for an overview, see Bermúdez, 1998, pp. 203-207). For example, Churchland (2012, p. 123) states that by a "map" one can understand a "high-dimensional structural homomorph of the objective similarity-and-difference structure of some objective, abstract feature-space." Cognitive maps are defined by the type or format of the information they "contain," as well as the structural organization of that information. Interestingly, Churchland (2012) continues by stating that "then presumably its virtues and vices, and the procedures for determining them, will parallel the comparatively prosaic case of a geographical map" (p. 123). The

\footnotetext{
${ }^{2}$ The idea of model-based learning (introduced by Tolman, 1984) as a contrastive mechanism to 'habitual responses' (Thorndike, 1898) is characterized by 'goal-directed actions' that are purposefully selected in order to achieve a particular state of the affairs. In the case of classical conditioning, the organism is a passive perceiver, while instrumental conditioning reflects the organism's ability to actively use the associative knowledge it obtains (and, through this, test and refine this knowledge). In other words, while "stimulus-driven control can be thought of as retrospective in that it depends on integrating past experience, goal-directed control may be thought of as prospective in that it leverages a cognitive map of the decision problem to flexibly revalue states and actions" (O’Doherty, Cockburn, \& Pauli, 2017, p. 76). This cognitive map is commonly understood to have spatial structure in which locations are not represented only in terms of their reward-related values, but the space is represented as open, facilitating exploration and adaptation of routes taken by the animal. This representation of space it taken to be a result of incidental or latent learning and is a simple 'by-product' of explora tion independent of reinforcement (Doeller \& Burgess, 2008).

${ }^{3}$ At this point of time, how this brain function was implemented could not be addressed due to a lack of techniques to record brain activity in freely moving animals. Strumwasser (1958) made approaching this question possible by pioneering the technique of implanting microwires.

${ }^{4}$ More recently, Rescorla (2009) proposed a differentiation between cognitive maps in three senses: trivial, loose and strict. Churchland's above characterization is closest to a loose understanding of cognitive maps, in as much as it can be read to assume the isomorphism to be of a geometrical, affine or metric kind. However, this does not need to be the case and Rescorla's treatment of cognitive maps cannot easily accommodate the approach presented in this paper. The main reason for this is that any purely geometric treatment of maps is a-temporal, while the understanding of cognitive maps and imagery presented here is based on spatio-temporal continuity.
} 
tendency to compare cognitive maps with geographical maps bears diagnostical similarity with the pictorial treatment that mental imagery has and continues to receive. Arguably, the reason for the attractiveness of this idea lies in the intuitive appeal of characterizing percepts in terms of their "resemblance" to external objects (an idea that can be traced back to Plato, Hume and Locke, amongst others). However, using this intuitive and simplistic understanding of imagery and the nature of the spatial component in imagistic cognition is hindering in terms of understanding the role of mental imagery in cognition.

The parallel between cognitive maps and mental imagery is not a novel one and the principle reason for this is that both are grounded in a problematic approach to spatial relationships, i.e. to the way the brain codes spatial information. Kosslyn's influential quasipictorial theory was motivated by demonstrations of various spatial isomorphisms between stimuli and its mental representation. It was demonstrated by Shepard and colleagues (Shepard \& Cooper, 1986; Shepard \& Metzler, 1971) that the time required to rotate a mental image correlated with the size of the angle of the rotation required. This was taken as evidence that mental images are formed and then rotated in the mind through a process that is isomorphic in time and space to the rotation of real presented objects. Furthermore, the time required to attend to different image features was shown to be correlated with the spatial distance between them on the presented image (Kosslyn, 1973, 1980). In other words, the size of the formed mental image influences the time needed to access the details of the image. These demonstrations of the spatial features of the format in which the presented stimuli were represented were taken as a strong argument for the particular nature of visual imagery that is different from propositional encodings.

Kosslyn argued that such representations are maintained in the "visual buffer," which functions as a $2 \mathrm{D}$ coordinate space that is "not an actual physical one but is rather a functional space defined by the way processes access the structure" (Kosslyn, 1981, p. 49). Kosslyn subscribes to the Kantian approach by claiming that "the organization of the visual buffer is innately determined and fixed" (p. 49). When it comes to accessibility, the contents of the visual buffer are transient in nature and decay rather quickly, and "information is represented by selectively activating local regions of the space" (p. 49). ${ }^{5}$ The format of the data structure supported by the visual buffer establishes a mapping between the represented object and the representational unit with respect to "size, shape, orientation, and location information" (p. 49). Therefore, "surface images consist of regions of activation in the visual buffer that correspond to regions of depicted objects, with distances between the regions on an object (as seen from a particular point of view) being preserved by distances between the regions used to represent it in the medium. Importantly, distance in the medium can be defined without reference to actual physical distance but merely in terms of the number of locations intervening between any two locations" (p. 50). In other words, any attempt to measure this "space" directly by measuring the metric units between

\footnotetext{
${ }^{5}$ The formatting of the visual buffer is inspired by retinotopic maps, while its accessibility is modelled on the differences between the foveal and peripheral vision.
} 
the parts of the image is futile because the measurements are in an important sense "functional". It is hard to pinpoint what is exactly being claimed here, but since we are not dealing with a pictorial representation in the classical Aristotelian sense, interpretation of the measurements in a functionalist metric system is needed to make sense of the data structures held within the visual buffer.

While accepting that such a functionalist representational space might be seen as attractive, this suffers from the problem of ascribing all the desired characteristics of the representational vehicles of investigation (mental imagery, in this case) to a purported "blackboard" which contains these objects. Without additional treatment of the blackboard itself, the question of what makes the imagery representational vehicles sui generis remains unanswered. Nevertheless, Kosslyn's and Shepherd's demonstrations show that whatever the characteristics of the underlying mental operations are, they do produce behavior that is in line with the spatio-temporal characteristics of the physical environment. ${ }^{6}$ "Mental imagery" as a term was suggested as a label for a sui generis format of information storage that is necessary for the working of a mental operation or mechanism such as mental rotation. In other words, certain operations can be carried out on the representational format of mental imagery that cannot be carried out on a different format. Due to the precise mapping to the physical characteristics of the stimulus (i.e. angle of rotation or physical distance), this novel format was assumed to have a spatial dimension (to which it owes its various names, e.g. mental imagery, quasi-pictorial representations, imagistic representations etc.). However, the exact relationship between physical space and the space-like characteristics of mental imagery have received little attention.

In order to understand what the features are that make mental imagery a sui generis form of mental representation and to assess their functional role in cognition, the spatio-temporal organizing principles of the informational structures that are mental images need to be understood. Early suggestions by Kosslyn and colleagues of the metric homomorphism between physical space and representational space are a good starting point but are not a clear characterization of mental imagery as a type of representational format. Additionally, this relatively simplistic understanding of the spatial component in mental imagery leads to a narrow understanding of the similarities between cognitive maps and mental imagery. In fact, the scientific understanding of cognitive maps has been continually developing and-as will become clear in the following section-these advances suggest the much

\footnotetext{
${ }^{6}$ This, by itself, is a very interesting finding which seems prima facie at odds with the classical propositionalist treatment of mental processes. Additionally, many have and continue to see mental imagery as a challenge to functionalist computationalism per se. For example, Bermúdez (2014, p. 43) states that since all forms of information in a digital computer are represented via binary code, and each binary numeral caries a single unit of information, "the length of time it takes to process a piece of information is typically a function only of the quantity of information (the number of bits that are required to encode it). The particular information that is encoded ought not to matter." Thus, according to Bermúdez, "what the mental rotation experiments have been taken by many to show is that there are information processing tasks that take varying amounts of time even though the quantity of information remains the same" (p. 43). Mental rotation shows that the way information is structured matters, not just the bit-size of the information.
} 
more complex role that space and time have to play in structuring mental content. The next section offers an overview of the most important findings concerning the neural underpinnings of representing space.

\section{Neural Underpinnings of Representing Space: The Role of the Hippocampal Formation}

The hippocampus is a region that features prominently in brain research. The medial temporal lobes generally, and the hippocampal formation ${ }^{7}$ in particular, have long been implicated in episodic memory formation processes (N. J. Cohen \& Squire, 1980; Scoville $\&$ Milner, 1957). As an attempt to treat his severe epilepsy, Henry Molaison (HM) underwent a bilateral medial temporal lobectomy procedure in which two thirds of his hippocampi, parahippocampal cortices, entorhinal cortices, piriform cortices and amygdalae were removed. As a consequence, HM suffered severe anterograde amnesia and was unable to form new semantic or episodic self-experienced memories. ${ }^{8}$ This extremely welldocumented case resulted in a pervasive understanding of the hippocampal complex as being a "seat" of memory in the brain. A separate line of investigation was initiated by the discovery of place cells in the hippocampus, drawing attention to the role of the hippocampal formation in spatial memory and navigation (O'Keefe \& Dostrovsky, 1971; O'Keefe \& Nadel, 1978). Subsequent experiments (Cohen, LaRoche, \& Beharry, 1971; Morris, Garrud, Rawlins, \& O'Keefe, 1982; Olton, Walker, Gage, \& Johnson, 1977) were able to establish aspects of navigation that are particularly sensitive to hippocampal damage: navigation to an unmarked location from a variable starting point (i.e. Tolman's task), and navigation that includes memory (and avoidance) of previously visited locations.

As the investigations into the functional role of the hippocampus in both memory-related processes and spatial navigation are well established yet hard to reconcile, they have been developed in a largely independent fashion. However, with recent findings a framework that accounts for both lines of research has started to emerge. Several groups have been able to show that hippocampus neurons also respond to non-spatial features, such as odor (Eichenbaum, Kuperstein, Fagan, \& Nagode, 1987; Igarashi, Lu, Colgin, Moser, \& Moser, 2014;

\footnotetext{
${ }^{7}$ The hippocampal formation is a compound structure in the medial temporal lobe, whose gross structural characteristics are shared by all mammals. There is no consensus as to what structural elements are part of the hippocampal formation. This paper understands the hippocampal formation to be comprised of the dentate gyrus, the hippocampus, the presubiculum, subiculum, parasubiculum and the entorhinal cortex.

${ }^{8}$ Tulving (1984) suggested a 'snapshot view' of episodic memory which "focuses on conditions that bring about a slice of experience frozen in time which we identify as 'remembering'. The recursive operation [...] produces many snapshots whose orderly succession can create the mnemonic illusion of the flow of past time" (p. 231). However, one does not need to subscribe to this characterization. In this paper, the assumed nature of episodic memory follows the account of Hasselmo (2011) in characterizing episodic memory as a spatiotemporal trajectory where "the process of mental time travel goes beyond forming associations of single items with a single, static behavioral context of location and time and involves encoding of continuous segments of a spatiotemporal trajectory" (p. 3). As will become clear, this is a subtle yet important difference.
} 
Wood, Dudchenko, \& Eichenbaum, 1999), tactile information (Young, Fox, \& Eichenbaum, 1994), and timing (Hampson, Heyser, \& Deadwyler, 1993). These discoveries, together with the role of grid cells in path integration (which will be discussed in the following subsections), have begun to inspire a novel view on the functional role of the hippocampal formation in encoding spatio-temporal information.

When recording from rats moving freely in a bounded area, O'Keefe \& Dostrovsky (1971) first reported on individual hippocampal cells being active only when an animal was in a particular place in the enclosure - their "place field." Place cells are not organized in a topographical manner, i.e. the place fields of neighboring place cells are not more similar than those of cells pairs farther away from each other (O'Keefe, 1976; Wilson \& McNaughton, 1994). ${ }^{9}$ A key finding was that the combination of cells active at each location was unique - the particular combination of active cells and their firing pattern was a "signature" of the environment the animal was exploring. This led O'Keefe \& Nadel (1978) to suggest that the hippocampus is the locus of a complex cognitive map of the environment - a result that finally fleshed out the implementation of Tolman's (1948) behavioral results. ${ }^{10}$

Several decades later, Hafting and colleagues (2005) discovered a novel cell type in the entorhinal cortex ${ }^{11}$ that was in many ways similar to place cells: grid cells. Like place cells, grid cells were active in particular places in the enclosed environment; however, the activated cells together formed nodes of an extended triangular (or hexagonal) grid that tiled the entire environment, "like the cross-points of graph paper" (Moser \& Moser, 2007). Cells in the same local area of the entorhinal cortex fire with the same spacing (i.e. distance between active fields) and grid orientation (the tilt of the grid relative to a reference axis), but in a different spatial phase (displacement in the $\mathrm{x}$ and $\mathrm{y}$ directions relative to an external reference point; ibid.). Subsequent studies revealed that grid cells were part of a wider network of different cell types that encode particular spatial aspects of the environment, such as the head direction cells (first described in the dorsal presubiculum by Ranck, 1984; Sargolini et al., 2006), border and speed cells (Savelli, Yoganarasimha, \& Knierim, 2008), and, often, cells with a combined function (Solstad, Boccara, Kropff, Moser, \& Moser, 2008). Head direction cells are active when the head of an animal is oriented in a certain direction and provide compass-like directional information. Border cells are active in reference to the limits (such as walls or other obstacles) of the environment the animal encounters.

\footnotetext{
${ }^{9}$ However, the size of the place fields does increase in a topographic manner, from the dorsal to the ventral hippocampus (Jung, Wiener, \& McNaughton, 1994; Kjelstrup et al., 2008).

${ }^{10}$ The results of O'Keefe \& Dostrovsky have received significant attention in the philosophical community, specifically in the realm of establishing the origins or 'grounding' principles of representational content. It is argued that structural correspondence between the spatial features of the environment and the firing patterns in the hippocampus constitutes a necessary condition for representing the environment, in addition to the observable behavioral consequences of this mapping (as in the case of maze navigation). For a recent treatment of this issue see Shea (2018).

${ }^{11}$ The entorhinal cortex is part of the hippocampal complex and is the main interface between the hippocampus and the neocortex.
} 
The different functions of place cells, grid cells and head direction cells in particular have sparked an ongoing debate concerning what type of spatial information these cells represent, as well as how this information is combined to allow flexible spatio-temporal representations. An important distinction needs to be introduced at this point: the difference between allocentric and egocentric space. If spatial information is conveyed in an allocentric manner, no information regarding the subjective standpoint of the observer is used as an organizing principle, the opposite being the case for the egocentric representation of spatial information. This is to say that when retrieving spatial information in an allocentric space, all viewpoints are accessible with no priority given to the particular viewpoints from which a particular piece of information was encountered (i.e. if a rat runs into a piece of cheese in a maze, the location of the cheese will be remembered with regards to the whole maze and independent of the specific viewpoint or scene in which the cheese was originally encountered). With this in mind, there is a well-established understanding that grid cells make a major contribution to allocentric spatial representations by providing an underlying metric system for encoding spatial distances. On the other hand, head cells (and other proprioceptive signals) are vital for egocentric representations as they provide a subject-dependent viewpoint. However, the role of the hippocampus has remained somewhat of a mystery.

The first step in understanding the hippocampal contribution was to clearly demonstrate that population activity of hippocampal cells is not only unique for particular environments, but that an environment that has already been encountered will result in the same pattern of activation upon later encounters. Ferbinteanu \& Shapiro (2003) showed that hippocampal place cells encode not only the current environment in which the animal finds itself, but also previously encountered environments. More importantly, a single hippocampal cell can partake in the overall population representation of many different environments, with its activity constantly being adapted and remodeled, based on not just the characteristic of the environment itself but also on changes in motivational state and different testing conditions (Leutgeb et al., 2005; Markus et al., 1995). This simultaneous rearrangement of many place cells in different environments was called "remapping," and once learned it can remain stable (Lever, Wills, Cacucci, Burgess, \& O’Keefe, 2002). Therefore, hippocampal place cell activity is i) a unique "signature" of a specific environment and ii) coupled with that environment over time, whilst still able to accommodate slight changes in the environment. In other words, when exploring an environment, the hippocampus brings together the episodic memory of the exploration and the spatial representation (i.e. cognitive map) of the environment ${ }^{12}$. Thus, the role of the hippocampus seems to be an integrative one, i.e.

\footnotetext{
${ }^{12}$ There are additional demonstrations of the relationship between memory and space coding in the brain: after encoding, memories go through additional consolidation, most commonly during sleep. Wilson and McNaughton (1994) showed that the ordering in which the hippocampal neurons are activated during active navigation correspond to the sequence of activations during sleep (i.e. 'replay'). Recent work has suggested that similar mechanisms are at play not only in memory consolidation, but also in planning future behavior and 'mental time travel' through the cognitive map, in past and present (Suddendorf \& Corballis, 2007). Overlapping hippocampal networks are activated when participants are asked to recall an episode and imagine an event that has never taken
} 
allocentric information and egocentric information are combined into a representational format that allows flexible spatio-temporal encoding. Importantly, the hippocampus is not particular to a given cognitive function (e.g. episodic memory, spatial navigation and path finding or imagery) but is a structure that provides a specific type of spatio-temporal information that is widely used by the brain in all these tasks.

Thus, the information provided by the hippocampus underpins a wide array of cognitive processes, all of which rely on spatio-temporal information. Path integration has proved to be useful in order to better understand the structure and format of information represented by the hippocampus, which is of key importance when it comes to the flexibility with which the hippocampus can underpin a range of different cognitive processes. Path integration is the ability of animals to travel between locations via a straight path using no external information by "continuous integration of self-movement so that global vectors connecting past and present locations can be continuously updated" (Gil et al., 2018, manuscript). The existence of this landmark-independent navigational system in animals was first suggested by O'Keefe (1976) and was subsequently confirmed by Mittelstaedt \& Mittelstaedt (1980). ${ }^{13}$ Path integration is achieved by combining information from head direction neurons and speed neurons; however, this information has to be integrated in a manner that provides a metric system, i.e. a system relative to which the animal is performing the movement. There is accumulating evidence to suggest that this is the role of the entorhinal grid cells, the spacing and geometrical structure of which is independent of the characteristics of the environment, such as size and shape (Fyhn, Hafting, Treves, Moser, \& Moser, 2005; Hafting et al., 2005). In addition, the firing pattern of grid cells is constant in different environments (c.f. place cells), which is another clue that grid cells are not influenced by external sensory stimulation.

Together, the activity of this network of neurons is sensitive to various types of self-motion cues and is referenced to an underlying grid cell network, thereby creating a metric representation of the environment. In other words, "as an animal moves through its environment, the location-specific activity in the grid cell network is probably updated principally by a path integration-based mechanism. The spatial code is therefore a relative one in the sense that the firing of one set of cells is determined by the preceding activity state of the network and the distance and direction moved by the animal in the intervening time, and is not determined directly by the pattern of environmental stimuli received by an animal at a given location” (McNaughton, Battaglia, Jensen, Moser, \& Moser, 2006, p. 668).

place (Hassabis, Kumaran, \& Maguire, 2007). Additionally, (Hassabis, Kumaran, Vann, \& Maguire, 2007) reported that patients with hippocampal amnesia are unable to imagine new experiences.

${ }^{13}$ There are several competing models regarding the implementation of path integration in the neural substrate, the continuous attractor model being particularly influential (initially suggested by Tsodyks and Sejnowski (1995). For a detailed account concerning the current progress of the model see McNaughton, Battaglia, Jensen, Moser, \& Moser (2006). 
As originally suggested by (O'Keefe, 1976) and confirming Kant's idea, the grid cells seem to be an implementation of a universal space metric system that is independent of external stimulation, but is specific to the individual animal. ${ }^{14}$

How does the activity of this network of various types of cells involved in providing a universal spatio-temporal metric system influence the activity of the hippocampus? There is evidence that the activity of place cells is coupled to voluntary motion and, very early on, Vanderwolf (1969) and Whishaw \& Vanderwolf (1973) showed that the oscillatory theta activity in hippocampal cells is determined by an animal's moving velocity. Therefore, "the scale of hippocampal place fields might be determined by a movement-speed signal that is generated outside the hippocampus through a summation of components related to ambulation, vestibular activation and optic flow" (McNaughton et al., 2006, p. 669). On the other hand, the influence of grid cells on place cells is an open issue, with several competing accounts. Overall, while the exact influence of different types of spatiotemporal information on hippocampal activity are not yet fully understood, much more is known about what kind of information modulates it. As has already been said, it is well established that the hippocampus is sensitive to the experiences an animal has had in a particular environment (e.g. odors and rewards encountered), not just to the objective features of the environment (such as its borders, light conditions etc.).

\section{Mental Imagery Reconsidered: \\ The Role of the Hippocampus in Representing Complex Spatial Structure}

The starting assumption of this work was in agreement with Kosslyn's approach to the problem of mental imagery: the defining feature of mental images is their relation to physical space. Kosslyn understood this relation to be a functional one, i.e. mental images are not 2D images or wax imprints of the spatial structure of the environment, they are simply information formatted in a way that conveys visuo-spatial information to higher cognitive processes. ${ }^{15}$ Determining the functional role of spatial information has been at the center of research regarding the principles of coding spatial information in the hippocampal formation. Kosslyn takes mental rotation and scanning to be paradigmatic examples of mental imagery, but the line of investigation presented here (arguably starting with the work of O'Keefe, 1976) gives reason to think of mental imagery in wider terms as the underlying representational format of both cognitive maps as well as episodic memory. ${ }^{16}$ The strong

\footnotetext{
${ }^{14}$ If this is the case, a question about the origin of the grid system immediately arises. There is considerable evidence that path integration is dependent on an intact grid system (e.g., Gil et al., 2018; Parron \& Save, 2004); there are also results showing that the grid system is expressed immediately in a novel environment (Fyhn, Hafting, Treves, Moser, \& Moser, 2005; Hafting, Fyhn, Molden, Moser, \& Moser, 2005). The evidence available thus far points to the innate formation of the grid system, although there is no consensus on this issue (for a detailed analysis see McNaughton et al., 2006).

${ }^{15}$ This functionalist approach in many ways parallels the ideas Descartes proposed in his Optics (Descartes, 1985).

${ }^{16}$ Rotation and scanning might simply be instances of these wider processes. In fact, in what follows I will argue that both cognitive maps and episodic memory are a result of the same underlying cognitive process.
} 
parallels between these cognitive processes that have traditionally been treated separately stems from the increased understanding of the role the hippocampus plays in all cognitive processes that rely on spatio-temporal information. In this section I will provide a theoretical treatment of these findings which will result in a finer characterization of mental imagery, both in terms of its information-bearing structure and its pervasiveness in cognition.

\subsection{The Hippocampus and Spatio-Temporal Chunking}

Trying to understand the organization of information in the hippocampus and its functional role in cognition, Eichenbaum \& Cohen (2014) made an influential suggestion according to which the hippocampus has two roles: first, it registers spatio-temporal discontinuities and thus performs "chunking" of spatio-temporal information; second, it "glues" the continuous stream of input into a unitary summary-like informational bin (i.e. creating a spatially coherent episode). In a similar vein, Hasselmo (2011) understands the role of the hippocampus as a navigational system through continuous spatio-temporal trajectories (i.e. instances of episodic memory). McNaughton and colleagues (2006, pp. 670-671) emphasize that "a crucial step in encoding a new episodic memory is the minimization of similarities between the new representation and representations that already exist in the network," as this process is what gives structure to experience. They state that the process of remapping, "observed after changes in a subset of the sensory cues (such as the geometrical shape or color of the test chamber) in an otherwise constant environment" might play an important role in this "through a pattern separation process, whereby small differences in cortical input patterns are amplified as they propagate through the hippocampal network, creating differences in the locations and/or firing rates for place fields." Buzsáki and Moser (2013) explicitly state that both finding a path through an environment and representing sequences in memory rely on the same mechanism: chunking. They claim that

chunking of the neuronal representation at each entry point can be assisted by the resetting of the path integrator or of the self-organized cell assembly trajectories by a new initial condition. Chunking is an efficient way to limit the accumulation of errors inherent in long sequences and is a frequent strategy for encoding episodic information. (Buzsáki and Moser, 2013, p. 6).

All these accounts of the functional role of the hippocampus share a common underlying understanding: the role of the hippocampus is to separate the incoming information into continuous chunks, thereby maximizing their dissimilarity in spatio-temporal terms. Another way to look at this is to say that the hippocampus is sensitive to the borders of maximum spatio-temporal discontinuity in the incoming stream of information (mostly from the entorhinal cortex); following this, it "normalizes" the chunks through their spatio-temporal dimension, in effect making them "smoother" in terms of their spatial and temporal derivatives. At first glance this approach might look similar to the traditional picture-like understanding of spatial representation that inspired early work on cognitive maps and mental imagery, as well as their apparent superficial similarities. However, the distinctive feature of these accounts that cannot be understated is that the chunks are spatio-temporal, 
and not just spatial. Taking this seriously amounts to rejecting the understanding of cognitive processes as unfolding in terms of any kind of picture-like representational formats. Space and time cannot be untangled and thus a classical understanding of cognitive maps and mental images as a-temporal units frozen in time is misleading. Moreover (as emphasized by Eichenbaum \& Cohen), the role of the hippocampus is not simply to separate the units into chunks, but also to establish a relationship between these units, i.e. to "bind" them in terms of their spatio-temporal relationships. This notion is easiest to understand when thinking about episodic memory, since it is introspectively a recalling into one's mind's eye of different spatio-temporal episodes that are connected with a varying degree of "certainty" (e.g. recalling the walk on the beach last Sunday or a concert one attended). In other words, there are episodes within episodes in any recall process which are connected to a varying degree.

However, it remains to be seen how this process relates to and supports imagining future scenarios and mental imagery more generally. In other words, how does chunking information in spatio-temporal bins relate to imagining a scenario or scene that was never experienced, as opposed to recalling a specific past event? It is clear that imagining anything must in some way rely on prior experience as it would be hard to conceive how one would be able to hold a cat on the mat in their minds eye if they had they never encountered a cat or a mat. ${ }^{17}$ In one way or another, what we imagine must be based on what we have encountered, which determines the "boundaries" of what we can imagine in the first place. Finding this boundary, and thus relating the past and the future, is in no way an easy endeavor; however, most of the research into this issue has been focused on the role of the hippocampus. The interest was sparked by some rather surprising findings which showed that the hippocampus is more activated when imagining the future than when remembering the past (Addis \& Schacter, 2012; Addis, Wong, \& Schacter, 2007; Buckner, 2010; Schacter \& Addis, 2009). This resulted in a number of experiments that tried to tease out exactly what the role of hippocampus is in imagining novel scenes and scenarios. However, the debate is still ongoing and important advances have been made.

One of the most prominent suggestions was made by Hassabis \& Maguire (2007), who put forward an influential and well-received idea about the functional role of the hippocampus which is in line with previous suggestions presented in this paper. According to these authors, the principal role of the hippocampus is to establish spatio-temporal relationships between the distinct elements that comprise a scene or a scenario, i.e. the hippocampus is the primary site of scene construction. Thus, the activity of the hippocampus is not only relevant for memory encoding but is also a key factor when elements of memories are actively recombined within a single spatio-temporal frame in order to create novel

\footnotetext{
${ }^{17}$ Interestingly — and this is where the hippocampus will turn out to play a key role - it is a priori much less problematic that a person might be able to imagine these two objects in a particular spatial relationship (i.e. the cat lying on the mat) without them ever seeing a cat lying on a mat beforehand. This asymmetry is rather telling, and I will come back to it shortly.
} 
mental content, i.e. imagery. ${ }^{18}$ The next section highlights how this process of scene construction — or "relational binding," as it has been called by (Olsen, Moses, Riggs, \& Ryan, 2012) — underlies mental imagery.

\subsection{The Hippocampus and Scene Construction}

This section attempts to provide a more detailed description of the functional role of the hippocampus in processing complex spatial structures, which will in turn shed light on the nature of the "chunks" of information that are processed by the hippocampus and their role in mental imagery. A complex visual scene contains several or many visual elements that have a specific spatial relationship to each other, and this organization or structure is important for future adaptive use. In other words, under such conditions representing the relationship between elements - not just individual elements themselves - is important. One can take this idea a step further and argue that these two types of information are, in fact, intertwined in a way that means that they either cannot be separated, or become significantly less useful when separated. In other words, one can say that perceiving spatial relations between objects is part and parcel with perceiving the objects themselves (without a significant loss of quality).

Evidence for this comes from research into hemispatial neglect, a neurophysiological condition in which, due to brain damage, a deficit in attention to and awareness of one side of the visual field is present. Patients are often unable to orient themselves in their environment and cannot detect objects on the affected side, although they are not blind to the stimuli presented (e.g. they are successful in detecting highly salient items). ${ }^{19}$ A particularly telling line of evidence comes from visual search experiments: patients who are unable to find objects in a cluttered scene are nevertheless able to find them when they are presented in isolation (Eglin, Robertson, \& Knight, 1989; Husain et al., 2001). This is evidence that individual objects on the neglected side are represented and can be accessed, but they are not successfully integrated in their context. Furthermore, many patients repeatedly re-fixate the same items in the visual search; this indicates deficiencies in visual working memory, which is necessary for saccadic integration (which is a non-lateralized process). The combination of impairments observed in hemispatial neglect, namely the

\footnotetext{
${ }^{18}$ Importantly, this process is a process of relating memory elements within space and time (e.g. positioning the cat over the mat) and not a process of using verbal or semantic information in a non-pictorial or non-spatial manner. According to the authors, this distinction is important and a lot of disagreement about the functional role of the hippocampus in mental imagery stems from the fact that many experiments do not carefully control the nature of the information participants are expected to use when creating scenes, nor the spatial coherence of the resulting scene, which is the most important contribution of the hippocampus.

${ }^{19}$ In a classical demonstration of the impairments, (Bisiach \& Luzzatti, 1978) asked neglect patients from Milan to imagine they were standing on the steps of the cathedral on the city's main square and draw what they were 'seeing'. Patients failed to report items located in space contralateral to the damaged side of their brain. However, when asked to turn around and report on what they saw, they failed to report the items they had previously reported.
} 
difficulty in saccadic integration of spatial information and in detecting or imagining objects in context, is an important clue in trying to understand the functional role that the encoding of physical spatial relations plays in cognition. If an object is presented in context but its spatial relationship with the contextual elements is not available, the object will most likely go undetected. On the other hand, spatio-temporal continuity (e.g. saccade integration) plays a crucial role in object-related memory formation. Both of these patterns of impairment point in the same direction: when an object is presented within a spatiotemporal continuum (i.e. context), it cannot be accessed independently of that context in pathological situations when the relation between the object and its context is either not coded successfully (by integrating self-movement cues such as saccadic eye movements) or cannot be accessed. ${ }^{20}$

Thus, if we understand object representations as constructs based on spatio-temporal continuity, these constructs are part of a relational structure and cannot be coded and accessed on their own. This underlying relational structure is, arguably, based on spatio-temporal continuity. An object embedded in context cannot be accessed on its own as its representation is entangled within the spatio-temporal structure of the scene. Thus, the case of hemispatial neglect is particularly interesting as it shows very clearly that perceiving the world around us relies in a very fundamental way on perceiving the spatio-temporal continuity between the individual elements of a scene. If this is the case in perceiving a scene, one might parsimoniously argue that it is also the case in remembering it or imagining a novel scene or scenario, i.e. elements of the scene are represented through "relational binding" with other elements and their context. Indeed, the role of the hippocampus seems to be to support this relationship. In recent years, this view has been supported by steadily accumulating evidence for the involvement of the hippocampus in perceiving complex objects and scenes at the earliest stages of visual processing (for a review see Lee, Yeung, \& Barense, 2012).

To conclude, the hippocampus brings together the subjective experience of the environment perceived by an organism with a representation of the environment in a universal metric-code that is not specific to the particular environment due to the involvement of grid cells. Notably, this process also uses information provided by the proprioceptive system, most prominently the head cells. This results in a complex representation of the environment that is not simply due to the "sensory input impinging" on the organism but is "a more abstract concept of a place, [...] a mental representation of where the rat 'thinks' it is" (Bird, Bisby, \& Burgess, 2012, p. 3). How exactly is the hippocampus suggested to support mental imagery? Essentially, as hinted at previously, its contribution is twofold. First, and stemming from the parallel role the hippocampus plays in memory encoding, "a partial cue concerning the surrounding boundaries and visual textures of a familiar environment can cause reactivation of the complete place cell representation corresponding to

\footnotetext{
${ }^{20}$ However, object perception on its own is intact. For example, increasing the salience of the object within its context will often result in successful detection. This might be an instance of the separation between the dorsal and ventral streams in visual processing.
} 
a single location" (p. 3). In other words, due to previous encounters with similar environments, the hippocampus is able to facilitate reactivation of a wide range of brain regions by use of various different types of cuing information (e.g. scent, previous reward, particular type of behavior previously engaged in, etc.). In this way, the hippocampus facilitates reactivation by similarity - a process that results in novel spatio-temporal chunks that are formed on the basis of previous experience that is registered or maintained by the hippocampus. Second, the hippocampus is a "locus" of "the formation of associations between disparate elements of an episode, occurring across space and time and in the flexible manipulation of learned relationships among items (the relational memory theory)" (Bird et al., 2012, p. 4, see also Eichenbaum, 1993; Olsen et al., 2012; Zeithamova, Schlichting, \& Preston, 2012). In other words, the hippocampus is able to bring together and bind various different elements in a coherent, novel and unique spatio-temporal frame. This is the underlying mechanism that is necessary not just for mental imagery, but also for forward projection and planning. Therefore, "the hippocampus is required to form mental images of scenes that are spatially coherent rather than simply stereotyped lists of the content of a scene" (Bird et al., 2012, p. 4). ${ }^{21}$

Following the line of evidence presented in this paper, we should radically reshape our traditional understanding of mental images as a-temporal picture-like units of cognition and start to view them as complex representational elements that are separated out of the continuous flow of information on the basis of their uniform character in space and time. Under this interpretation, mental imagery has an underlying spatial and temporal dimension which is uniform and "untangled" enough to make the phenomenological aspect of it seem snapshot-like. However, this spatio-temporal dimension is the "access point" which allows a continuous flow of an imagistic episode (such as in mental rotation, scanning, path finding, reliving episodes from the past or envisaging future events). These spatial imagery processes depend on the functioning of the hippocampus, first in terms of separating or "grouping" the coherent elements of the incoming stream of information, and then in allowing the recombination and reuse of these elements in order to construct novel mental images.

\section{Conclusion}

The aim of this paper was to suggest a novel account of mental imagery according to which mental images are not a-temporal picture-like representations, but processes characterized by their spatio-temporal continuity. Evidence based on recent advances in understanding the functional role of the hippocampal formation in cognition and spatial coding in particular has been provided. The key role in providing a spatial metric system is played by the interaction of grid cells found in the entorhinal cortex, with several other types of cells

\footnotetext{
${ }^{21}$ The authors additionally suggest that this spatio-temporal coherence or boundness is what provides vividness to mental images. In other words, a stronger bond between elements results in greater spatio-temporal detail which, in turns, gives rise to an increased sense of phenomenological vividness as well as to the ability to semantically access a greater amount of detail.
} 
providing information from the vestibular system. However, the encoding and access to mental imagery is assumed to take place in the hippocampus, facilitated by the information relayed by the entorhinal cortex. The hippocampus "chunks" the incoming stream of stimulation into continuous spatio-temporal trajectories, or mental images. Additionally, the elements formed due to prior experiences are recombined and complex relationships between them are established in the process of "scene reconstruction" that underpins all mental imagery processes. Under this account, mental images are a pervasive form of cognition that is supported by the complex interaction of the hippocampus and the entorhinal cortex, encompassing cognitive functions such as navigation, episodic memory, as well as mental rotation and scanning.

\section{Acknowledgements}

This work was supported by the Croatian Science Foundation under the project number 5343.

\section{References}

Addis, D. R., \& Schacter, D. (2012). The hippocampus and imagining the future: Where do we stand? Frontiers in Human Neuroscience, 5, 173. https://doi.org/10.3389/fnhum.2011.00173

Addis, D. R., Wong, A. T., \& Schacter, D. L. (2007). Remembering the past and imagining the future: common and distinct neural substrates during event construction and elaboration. Neuropsychologia, 45(7), 1363-1377. https://doi.org/10.1016/j.neuropsychologia.2006.10.016

Barsalou, L. W. (1999). Perceptions of perceptual symbols. Behavioral and Brain Sciences, 22(4), 637-660. https://doi.org/10.1017/S0140525X99532147

Bermúdez, J. L. (1998). The paradox of self-consciousness. Cambridge, MA: MIT Press. https://doi.org/10.7551/mitpress/5227.001.0001

Bermúdez, J. L. (2014). Cognitive science: An introduction to the science of the mind. Cambridge University Press. https://doi.org/10.1017/CBO9781107279889

Bird, C. M., Bisby, J. A., \& Burgess, N. (2012). The hippocampus and spatial constraints on mental imagery. Frontiers in Human Neuroscience, 6, 142. https://doi.org/10.3389/fnhum.2012.00142

Bisiach, E., \& Luzzatti, C. (1978). Unilateral neglect of representational space. Cortex, 14(1), 129-133. https://doi.org/10.1016/S0010-9452(78)80016-1

Buckner, R. L. (2010). The role of the hippocampus in prediction and imagination. Annual Review of Psychology, 61, 27-48. https://doi.org/10.1146/annurev.psych.60.110707.163508

Burgess, N., Maguire, E. A., \& O'Keefe, J. (2002). The Human Hippocampus and Spatial and Episodic Memory. Neuron, 35(4), 625-641. https://doi.org/10.1016/S0896-6273(02)00830-9

Buzsáki, G., \& Moser, E. I. (2013). Memory, navigation and theta rhythm in the hippocampal-entorhinal system. Nature Neuroscience, 16(2), 130. https://doi.org/10.1038/nn.3304 
Churchland, P. M. (2012). Plato's camera: How the physical brain captures a landscape of abstract universals. Cambridge, MA: MIT Press. https://doi.org/10.7551/mitpress/9116.001.0001

Cohen, J. S., LaRoche, J. P., \& Beharry, E. (1971). Response perseveration in the hippocampal lesioned rat. Psychonomic Science, 23(3), 221-223. https://doi.org/10.3758/BF03336086

Cohen, N. J., \& Squire, L. R. (1980). Preserved learning and retention of pattern-analyzing skill in amnesia: Dissociation of knowing how and knowing that. Science, 210(4466), 207-210. https://doi.org/10.1126/science.7414331

Descartes, R. (1985). Optics. In J. Cottingham, R. Stoothoff and D. Murdoch (Eds.), The Philosophical Writings of Descartes (Vol. 1). Cambridge, UK: Cambridge University Press. https://doi.org/10.1017/CBO9780511805042

Doeller, C. F., \& Burgess, N. (2008). Distinct error-correcting and incidental learning of location relative to landmarks and boundaries. Proceedings of the National Academy of Sciences, 105(15), 5909-5914. https://doi.org/10.1073/pnas.0711433105

Eglin, M., Robertson, L. C., \& Knight, R. T. (1989). Visual search performance in the neglect syndrome. Journal of Cognitive Neuroscience, 1(4), 372-385. https://doi.org/10.1162/jocn.1989.1.4.372

Eichenbaum, H., Kuperstein, M., Fagan, A., \& Nagode, J. (1987). Cue-sampling and goal-approach correlates of hippocampal unit activity in rats performing an odor-discrimination task. Journal of Neuroscience, 7(3), 716-732. https://doi.org/10.1523/JNEUROSCI.07-03-00716.1987

Eichenbaum, H. (1993). Memory, amnesia, and the hippocampal system. Cambridge, MA: MIT Press.

Eichenbaum, H., \& Cohen, N. J. (2014). Can we reconcile the declarative memory and spatial navigation views on hippocampal function? Neuron, 83(4), 764-770.

https://doi.org/10.1016/j.neuron.2014.07.032

Ferbinteanu, J., \& Shapiro, M. L. (2003). Prospective and retrospective memory coding in the hippocampus. Neuron, 40(6), 1227-1239. https://doi.org/10.1016/s0896-6273(03)00752-9

Fyhn, M., Hafting, T., Treves, A., Moser, M. B., \& Moser, E. I. (2005). Preserved spatial and temporal firing structure in entorhinal grid cells during remapping in the hippocampus. In Society for Neuroscience Abstracts (Vol. 198).

Gallistel, C. R. (1990). Learning, development, and conceptual change: The organization of learning. Cambridge, MA: MIT Press.

Gil, M., Ancau, M., Schlesiger, M. I., Neitz, A., Allen, K., Marco, R. J. D., \& Monyer, H. (2018). Impaired path integration in mice with disrupted grid cell firing. Nature Neuroscience, 21(1), 81-91. https://doi.org/10.1038/s41593-017-0039-3

Hafting, T., Fyhn, M., Molden, S., Moser, M.-B., \& Moser, E. I. (2005). Microstructure of a spatial map in the entorhinal cortex. Nature, 436(7052), 801. https://doi.org/10.1038/nature03721

Hampson, R. E., Heyser, C. J., \& Deadwyler, S. A. (1993). Hippocampal cell firing correlates of delayed-match-to-sample performance in the rat. Behavioral Neuroscience, 107(5), 715. https://doi.org/10.1037/0735-7044.107.5.715 
Hassabis, D., Kumaran, D., \& Maguire, E. A. (2007). Using imagination to understand the neural basis of episodic memory. Journal of Neuroscience, 27(52), 14365-14374.

https://doi.org/10.1523/JNEUROSCI.4549-07.2007

Hassabis, D., Kumaran, D., Vann, S. D., \& Maguire, E. A. (2007). Patients with hippocampal amnesia cannot imagine new experiences. Proceedings of the National Academy of Sciences, 104(5), 1726-1731. https://doi.org/10.1073/pnas.0610561104

Hassabis, D., \& Maguire, E. A. (2007). Deconstructing episodic memory with construction. Trends in Cognitive Sciences, 11(7), 299-306. https://doi.org/10.1016/j.tics.2007.05.001

Hasselmo, M. E. (2011). How we remember: Brain mechanisms of episodic memory. Cambridge, MA: MIT Press. https://doi.org/10.7551/mitpress/9780262016353.001.0001

Husain, M., Mannan, S., Hodgson, T., Wojciulik, E., Driver, J., \& Kennard, C. (2001). Impaired spatial working memory across saccades contributes to abnormal search in parietal neglect. Brain, 124(5), 941-952. https://doi.org/10.1093/brain/124.5.941

Igarashi, K. M., Lu, L., Colgin, L. L., Moser, M.-B., \& Moser, E. I. (2014). Coordination of entorhinal-hippocampal ensemble activity during associative learning. Nature, 510(7503), 143. https://doi.org/10.1038/nature13162

Jung, M. W., Wiener, S. I., \& McNaughton, B. L. (1994). Comparison of spatial firing characteristics of units in dorsal and ventral hippocampus of the rat. Journal of Neuroscience, 14(12), 7347-7356. https://doi.org/10.1523/JNEUROSCI.14-12-07347.1994

Kant, I. (1998). Critique of pure reason. Cambridge, UK: Cambridge University Press. (Original work published 1781) https://doi.org/10.1017/CBO9780511804649

Kjelstrup, K. B., Solstad, T., Brun, V. H., Hafting, T., Leutgeb, S., Witter, M. P., .. Moser, M.-B. (2008). Finite scale of spatial representation in the hippocampus. Science, 321(5885), 140-143. https://doi.org/10.1126/science.1157086

Kosslyn, S. M. (1981). The medium and the message in mental imagery: A theory. Psychological Review, 88(1), 46. https://doi.org/10.1037/0033-295X.88.1.46

Kosslyn, S. M. (1973). Scanning visual images: Some structural implications. Perception \& Psychophysics, 14(1), 90-94. https://doi.org/10.3758/BF03198621

Kosslyn, S. M. (1980). Image and mind. Cambridge, MA: Harvard University Press.

Lee, A. C., Yeung, L.-K., \& Barense, M. D. (2012). The hippocampus and visual perception. Frontiers in Human Neuroscience, 6, 91. https://doi.org/10.3389/fnhum.2012.00091

Leutgeb, S., Leutgeb, J. K., Barnes, C. A., Moser, E. I., McNaughton, B. L., \& Moser, M.-B. (2005). Independent codes for spatial and episodic memory in hippocampal neuronal ensembles. Science, 309(5734), 619-623. https://doi.org/10.1126/science.1114037

Lever, C., Wills, T., Cacucci, F., Burgess, N., \& O'keefe, J. (2002). Long-term plasticity in hippocampal place-cell representation of environmental geometry. Nature, 416(6876), 90. https://doi.org/10.1038/416090a 
Markus, E. J., Qin, Y.-L., Leonard, B., Skaggs, W. E., McNaughton, B. L., \& Barnes, C. A. (1995). Interactions between location and task affect the spatial and directional firing of hippocampal neurons. Journal of Neuroscience, 15(11), 7079-7094. https://doi.org/10.1523/JNEUROSCI.15-11-07079.1995

McNaughton, B. L., Battaglia, F. P., Jensen, O., Moser, E. I., \& Moser, M.-B. (2006). Path integration and the neural basis of the "cognitive map." Nature Reviews Neuroscience, 7(8), 663-678. https://doi.org/10.1038/nrn1932

Mittelstaedt, M.-L., \& Mittelstaedt, H. (1980). Homing by path integration in a mammal. Naturwissenschaften, 67(11), 566-567. https://doi.org/10.1007/BF00450672

Morris, R. G. M., Garrud, P., Rawlins, J. al, \& O'Keefe, J. (1982). Place navigation impaired in rats with hippocampal lesions. Nature, 297(5868), 681. https://doi.org/10.1038/297681a0

Moser, E., \& Moser, M.-B. (2007). Grid cells. Scholarpedia, 2(7), 3394. https://doi.org/10.4249/scholarpedia.3394

O’Doherty, J. P., Cockburn, J., \& Pauli, W. M. (2017). Learning, reward, and decision making. Annual Review of Psychology, 68, 73-100. https://doi.org/10.1146/annurev-psych-010416044216

O'Keefe, J. (1976). Place units in the hippocampus of the freely moving rat. Experimental Neurology, 51(1), 78-109. https://doi.org/10.1016/0014-4886(76)90055-8

O'Keefe, J., \& Dostrovsky, J. (1971). The hippocampus as a spatial map: Preliminary evidence from unit activity in the freely-moving rat. Brain Research. https://doi.org/10.1016/00068993(71)90358-1

O'Keefe, J., \& Nadel, L. (1978). The hippocampus as a cognitive map. Oxford, UK: Clarendon Press.

Olsen, R. K., Moses, S. N., Riggs, L., \& Ryan, J. D. (2012). The hippocampus supports multiple cognitive processes through relational binding and comparison. Frontiers in Human Neuroscience, 6, 146. https://doi.org/10.3389/fnhum.2012.00146

Olton, D. S., Walker, J. A., Gage, F. H., \& Johnson, C. T. (1977). Choice behavior of rats searching for food. Learning and Motivation, 8(3), 315-331. https://doi.org/10.1016/00239690(77)90055-8

Parron, C., \& Save, E. (2004). Evidence for entorhinal and parietal cortices involvement in path integration in the rat. Experimental Brain Research, 159(3), 349-359. https://doi.org/10.1007/s00221-004-1960-8

Prinz, J. J. (2004). Furnishing the mind: Concepts and their perceptual basis. Cambridge, MA: MIT Press.

Pylyshyn, Z. W. (1973). What the mind's eye tells the mind's brain: A critique of mental imagery. Psychological Bulletin, 80(1), 1. https://doi.org/10.1007/978-94-010-1193-8_1

Pylyshyn, Z. W. (1981). The imagery debate: Analogue media versus tacit knowledge. Psychological Review, 88(1), 16. https://doi.org/10.1037/0033-295x.88.1.16

Ranck Jr, J. B. (1984). Head-direction cells in the deep cell layers of dorsal presubiculum in freely moving rats. In Society for Neuroscience Abstracts (Vol. 10, p. 599). 
Sargolini, F., Fyhn, M., Hafting, T., McNaughton, B. L., Witter, M. P., Moser, M.-B., \& Moser, E. I. (2006). Conjunctive representation of position, direction, and velocity in entorhinal cortex. Science, 312(5774), 758-762. https://doi.org/10.1126/science.1125572

Savelli, F., Yoganarasimha, D., \& Knierim, J. J. (2008). Influence of boundary removal on the spatial representations of the medial entorhinal cortex. Hippocampus, 18(12), 1270-1282. https://doi.org/10.1002/hipo.20511

Schacter, D. L., \& Addis, D. R. (2009). On the nature of medial temporal lobe contributions to the constructive simulation of future events. Philosophical Transactions of the Royal Society of London B: Biological Sciences, 364(1521), 1245-1253. https://doi.org/10.1098/rstb.2008.0308

Scoville, W. B., \& Milner, B. (1957). Loss of recent memory after bilateral hippocampal lesions. Journal of Neurology, Neurosurgery, and Psychiatry, 20(1), 11.

https://doi.org/10.1136/jnnp.20.1.11

Shea, N. (2018). Representation in cognitive science. Oxford University Press. https://doi.org/10.1093/oso/9780198812883.001.0001

Shepard, R. N., \& Cooper, L. A. (1986). Mental images and their transformations. Cambridge. MA: The MIT Press.

Shepard, R. N., \& Metzler, J. (1971). Mental rotation of three-dimensional objects. Science, 171(3972), 701-703. https://doi.org/10.1126/science.171.3972.701

Solstad, T., Boccara, C. N., Kropff, E., Moser, M.-B., \& Moser, E. I. (2008). Representation of geometric borders in the entorhinal cortex. Science, 322(5909), 1865-1868. https://doi.org/10.1126/science.1166466

Strumwasser, F. (1958). Long-term recording from single neurons in brain of unrestrained mammals. Science, 127(3296), 469-470. https://doi.org/10.1126/science.127.3296.469

Suddendorf, T., \& Corballis, M. C. (2007). The evolution of foresight: What is mental time travel, and is it unique to humans? Behavioral and Brain Sciences, 30(3), 299-313. https://doi.org/10.1017/S0140525X07001975

Thorndike, E. L. (1898). Animal intelligence: an experimental study of the associative processes in animals. The Psychological Review: Monograph Supplements, 2(4), i. https://doi.org/10.1037/h0092987

Tolman, E. C. (1948). Cognitive maps in rats and men. Psychological Review, 55(4), 189. https://doi.org/10.1037/h0061626

Tolman, E. C., Ritchie, B. F., \& Kalish, D. (1946). Studies in spatial learning: Orientation and the short-cut. Journal of Experimental Psychology, 36(1), 13. https://doi.org/10.1037/h0053944

Tsodyks, M., \& Sejnowski, T. (1995). Associative memory and hippocampal place cells. International Journal of Neural Systems, 6, 81-86.

Tulving, E. (1984). Precis of elements of episodic memory. Behavioral and Brain Sciences, 7(2), 223-238. https://doi.org/10.1017/S0140525X0004440X

Vanderwolf, C. H. (1969). Hippocampal electrical activity and voluntary movement in the rat. Electroencephalography and Clinical Neurophysiology, 26(4), 407-418.

https://doi.org/10.1016/0013-4694(69)90092-3 
Whishaw, I. Q., \& Vanderwolf, C. H. (1973). Hippocampal EEG and behavior: change in amplitude and frequency of RSA (theta rhythm) associated with spontaneous and learned movement patterns in rats and cats. Behavioral Biology, 8(4), 461-484. https://doi.org/10.1016/S00916773(73)80041-0

Wilson, M. A., \& McNaughton, B. L. (1994). Reactivation of hippocampal ensemble memories during sleep. Science, 265(5172), 676-679. https://doi.org/10.1126/science.8036517

Wood, E. R., Dudchenko, P. A., \& Eichenbaum, H. (1999). The global record of memory in hippocampal neuronal activity. Nature, 397(6720), 613. https://doi.org/10.1038/17605

Young, B. J., Fox, G. D., \& Eichenbaum, H. (1994). Correlates of hippocampal complex-spike cell activity in rats performing a nonspatial radial maze task. Journal of Neuroscience, 14(11), 6553-6563. https://doi.org/10.1523/JNEUROSCI.14-11-06553.1994

Zeithamova, D., Schlichting, M. L., \& Preston, A. R. (2012). The hippocampus and inferential reasoning: building memories to navigate future decisions. Frontiers in Human Neuroscience, 6 , 70. https://doi.org/10.3389/fnhum.2012.00070

This publication has been prepared as part of the research grant "What is Thinking with Images?", SONATA 10, founded by the National Science Centre, Poland, on the basis of the decision No. 2015/19/D/HS1/02426. 